\title{
Human immunodeficiency virus-related Epstein-Barr virus-associated smooth muscle tumours: South African experience from Chris Hani Baragwanath Academic Hospital
}

\author{
Sugeshnee Pather ${ }^{\text {ade* }}$, Rosalind D. Wainwright ${ }^{\text {bde }}$, Faieza Sahidcde, Thembi Mashele ${ }^{\text {ade }}$, Eunice J. van den Berg ${ }^{\text {ade }}$ and Reena D. Mohanlalade $^{\text {and }}$ \\ ${ }^{a}$ Division of Anatomical Pathology, National Health Laboratory Service, Johannesburg, South Africa \\ ${ }^{b}$ Haematology and Oncology Unit, Department of Paediatrics, University of the Witwatersrand, Johannesburg, South Africa \\ 'Division of Infectious Diseases, Department of Internal Medicine, University of the Witwatersrand, Johannesburg, South Africa \\ ${ }^{d}$ Chris Hani Baragwanath Academic Hospital, Johannesburg, South Africa \\ ${ }^{e}$ Faculty of Health Sciences, University of the Witwatersrand, Johannesburg, South Africa \\ *Corresponding author, email. sugeshnee.pather@nhls.ac.za
}

Background: Despite the rampant human immunodeficiency (HIV) epidemic in Africa, there is a paucity of published data of HIV-related Epstein-Barr virus-associated smooth muscle tumours (EBV-SMT) from Africa.

Methods: We embarked on retrospective documentation of the clinicopathological features of confirmed HIV-related EBV-SMT over a 5-year time frame at the largest hospital in Africa. All haematoxylin and eosin stained tissue sections, immunohistochemistry and EBV in situ hybridisation (ISH) investigations were reviewed in conjunction with clinical data.

Results: Fourteen ( $n=14$ ) EBV-SMT were confirmed in 13 patients (age range: 10-53 years). Five paediatric patients and a predominance of females (70\%) were evident in this series. All patients were HIV seropositive and CD4 counts ranged from 1 to 1331 cells/ul (median 355 cells/ul; mean 442 cells/ul). Tumour-associated pain was a common symptom in the paediatric age group, while neurological symptoms were frequent in the adults due to paraspinal cervicothoracic involvement. Unusual topography, multifocality $(n=5)$ and smooth muscle morphology in association with round cell features $(n=3)$ were evident. Immunoexpression of desmin $(n=12)$, SMA $(n=12)$ and h-Caldesmon $(n=8)$ were consistent findings and positive EBV ISH nuclear signaling was demonstrated within all of these tumours. Treatment included antiretroviral therapy, surgical resection, radiation and/or palliative therapy.

Conclusion: HIV-associated EBV-SMT are rare tumours that may develop in paediatric or adult patients. A female predominance and multifocal topographic involvement may be evident. AIDS-related co-morbidities are likely to contribute to mortality; and when these tumours occur in paraspinal regions, debilitating neurological morbidity may manifest.

Keywords: Epstein-Barr virus, HIV, smooth muscle tumours, South Africa

\section{Introduction}

Epstein-Barr virus (EBV) is a common human gammaherpesvirus, and $90 \%$ (or more) of the human population worldwide are carriers thereof. ${ }^{1}$ EBV causes infectious mononucleosis and has an oncogenic influence in the development of non-Hodgkin lymphoma, classical Hodgkin lymphoma, post-transplantation associated lymphoproliferative disorders and carcinomas. ${ }^{2}$ Infrequently, EBV infection predisposes to the development of mesenchymal neoplasms of smooth muscle lineage which are known as EBV-associated smooth muscle tumours (EBV-SMT). These tumours have a specific propensity to develop in immunocompromised adult or paediatric patients who are transplant recipients, afflicted with congenital immunodeficiency disorders or human immunodeficiency virus (HIV) infected. ${ }^{3-5}$

EBV-SMT may manifest slightly more frequently in females patients, as solitary or multifocal lesions, within diverse topographic regions such as soft tissue, gastrointestinal tract, lung, central nervous system and skin, among other sites.,6 Although the exact pathogenesis of EBV-SMT remains elusive, researchers have suggested that it is via the cluster of differentiation 21 (CD21) receptor that EBV gains access to smooth muscle cells and promotes proliferation in HIV positive patients. ${ }^{4,6}$ It is noteworthy that increased expression of CD21 has not been consistently documented in EBV-SMT occurring in transplant recipients.
Despite the rampant epidemic of HIV infection in Africa, EBVSMT in South Africa is infrequently reported ${ }^{8-11}$ and the paucity of EBV-SMT data from Africa was noted in a review of published cases by Purgina et al. ${ }^{5}$ This article serves to contribute clinicopathological experience about HIV-related EBV-SMT in paediatric and adults patients at the largest hospital in Africa.

\section{Aim}

We aimed to retrospectively evaluate all HIV-related EBV-SMT that occurred in patients at a tertiary academic hospital in South Africa. Demographic factors, clinicopathological features, concomitant diseases, treatment and survival outcome were documented.

\section{Materials and method}

Systematised Nomenclature of Medicine (SNOMED) based searches of the laboratory datasets were performed to detect all cases of histopathologically-confirmed EBV-SMT from January 2010 to December 2014. Haematoxylin and eosin (H\&E) stained sections, immunohistochemistry and in situ hybridization investigations were routinely performed on these tumours for diagnostic purposes. Immunohistochemistry panel included desmin (Ventana, clone DE-R-11, prediluted at $5 \mu \mathrm{g} / \mathrm{ml}$ ), alpha smooth muscle actin (Ventana, clone 14A, prediluted at $0.02 \mu \mathrm{g} /$ $\mathrm{ml}$ ) and/or h-Caldesmon (Dako, clone h-CD, 1/600). 
EBV mRNA in situ hybridisation (Ventana Medical Systems, Inc INFORM EBER Probe, United States of America) was used to demonstrate latent infection by EBV. The probe hybridises to EBV-encoded RNA (EBER) transcripts in the nuclei of infected cells. The EBV probe is fluorescein labeled and a positive signal was seen as a blue-black nuclear stain.

The histopathology slides of all cases were reviewed by a histopathologist to confirm the diagnosis of EBV-SMT. The clinical information was retrieved from the patients' records by a paediatric oncologist and an infectious diseases specialist physician.

\section{Results}

Fourteen EBV-SMT ( $n=14$ ) were histopathologically confirmed in 13 patients and this series included 5 paediatric patients (Table 1). The age range was 10-53 years (mean $30 \mathrm{yrs}$; median $31 \mathrm{yrs}$ ) and a predominance of females was evident.

All the patients were HIV-seropositive as confirmed by enzymelinked immunosorbent assay. CD4 counts were documented within 1-3 mnths of the EBV-SMT diagnosis and ranged from 1 to 1331 cells/ul (mean 442 cells/ul; median 355 cells/ul; CD4 count reference range 500 - 2010 cells/ul).

Tumour-associated pain was a common symptom in the paediatric age group, while neurological symptoms were frequent in the adults due to intracranial or cervicothoracic paraspinal tumours. Five patients had radiological features of multifocal EBV-SMT (Table 1).

\section{Pathological features}

Smooth muscle morphology (Figure 1) was readily identified in 13 tumours and distinctive hypercellular primitive round cell features (Figure 2) were noted in 3 tumours. The average mitotic count ranged from $0-3 / 10$ high power fields; coagulative necrosis was present in 2 tumours and nuclear pleomorphism was evident in 2 tumours. Mild scattered lymphocytic

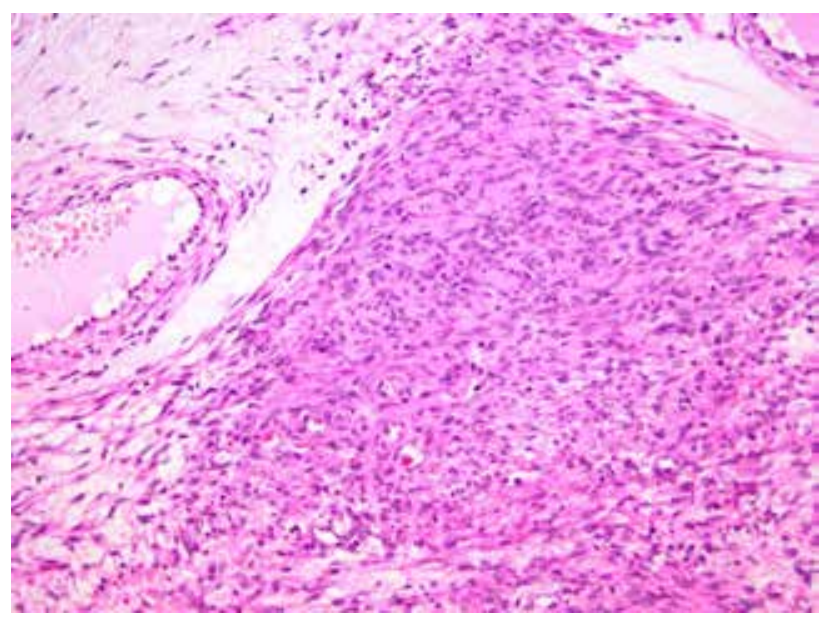

Figure 1: EBV-SMT comprising fascicles of smooth muscle (H\&E stain; X200 magnification).

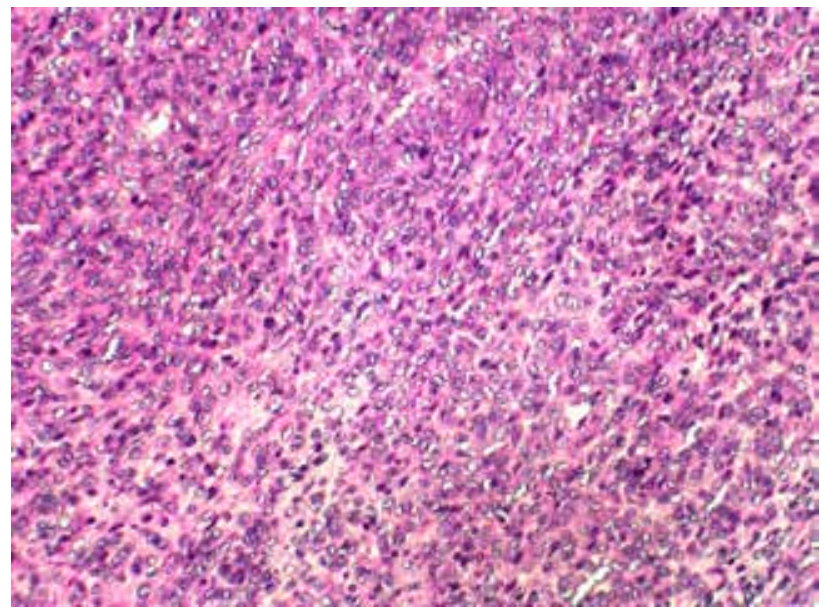

Figure 2: Hypercellular primitive round cell areas of EBV-SMT (H\&E stain X200)

Table 1: Salient clinical features of HIV-associated EBV-SMT.

\begin{tabular}{|c|c|c|c|c|c|c|c|}
\hline No. & Age (years) & Gender & CD4 (cells/uL) & Antiretroviral therapy* & Topographic site & Treatment & Survival outcome \\
\hline 1 & 13 & $\mathrm{~F}$ & 1 & Y & Paraspinal & Surgery & Demised $^{* *}$ \\
\hline 2 & 10 & $\mathrm{~F}$ & 902 & $\mathrm{Y}$ & Paraspinal \& intra-abdominal & Radiation & Alive \\
\hline 3 & 15 & $\mathrm{~F}$ & 616 & $\mathrm{Y}$ & Iris & Surgery & Alive \\
\hline 4 & 12 & $\mathrm{~F}$ & 1331 & $\mathrm{Y}$ & $\begin{array}{l}\text { Duodenal ampulla \& adrenal } \\
\text { gland }\end{array}$ & Palliation & Alive \\
\hline 5 & 12 & $\mathrm{~F}$ & 604 & Y & Paraspinal \& rib & Radiation & Alive ${ }^{* * *}$ \\
\hline 6 & 53 & M & 173 & Y & Intracranial & Surgery & Alive \\
\hline 7 & 45 & $\mathrm{~F}$ & 209 & $\mathrm{~N}$ & Paraspinal & Surgery \& Palliation & Alive (paraplegic) \\
\hline 8 & 37 & M & 27 & $\mathrm{~N}$ & Intracranial & Surgery & Demised $^{\circ}$ \\
\hline 9 & 43 & $\mathrm{~F}$ & 355 & Y & Soft tissue (hand) $\&$ paraspinal ${ }^{\circ \circ}$ & Palliation & Alive (paraplegic) \\
\hline 10 & 53 & $\mathrm{~F}$ & NIA & NIA & Soft tissue (thigh) & NIA & NIA \\
\hline 11 & 28 & $\mathrm{~F}$ & 574 & NIA & Paraspinal \& soft tissue (neck) & NIA & NIA \\
\hline 12 & 32 & $\mathrm{~F}$ & NIA & NIA & Duodenal ampulla & NIA & NIA \\
\hline 13 & 31 & M & 74 & $\mathrm{Y}$ & Paraspinal & NIA & NIA \\
\hline
\end{tabular}

Notes: $\mathrm{Y}$ - yes; $\mathrm{N}$ - no; NIA - no information available.

* Received prior to the diagnosis of EBV-SMT.

**Demised from sepsis 5 months after EBV-SMT diagnosis.

***Subsequently lost to follow up.

${ }^{\circ}$ Demised of pneumonia three years after EBV-SMT diagnosis; previously published case report. ${ }^{9}$

${ }^{\circ}$ Histopathologically confirmed multifocal EBV-SMT. 
inflammatory infiltrate was present in 10 tumours and moderateintensity lymphocytic infiltrate was noted in 4 tumours.

Immunohistochemistry was integral in confirming the smooth muscle histogenesis of these neoplasms. Desmin immunoreactivity occurred diffusely in $>50 \%$ of cells in 5 tumours, weak and focal staining was present in 7 tumours and negative staining was evident in 2 tumours. Smooth muscle actin (SMA) and $\mathrm{h}$-Caldesmon expression (Figure 3 ) were evident in 12 tumours (of 13 tested) and 8 tumours (of 8 tested), respectively.

Positive nuclear signaling using EBER ISH was demonstrated within all EBV-SMT reported herein. The proportion of tumour cells displaying positive signaling was variable and ranged from $10 \%$ to $90 \%$ (Figure 4 ).

Concomitant infection by cytomegalovirus was incidentally documented in the ampullary region of a paediatric patient who had clinical features of CMV retinitis, blindness in the right eye and left pulmonary tuberculous destruction.

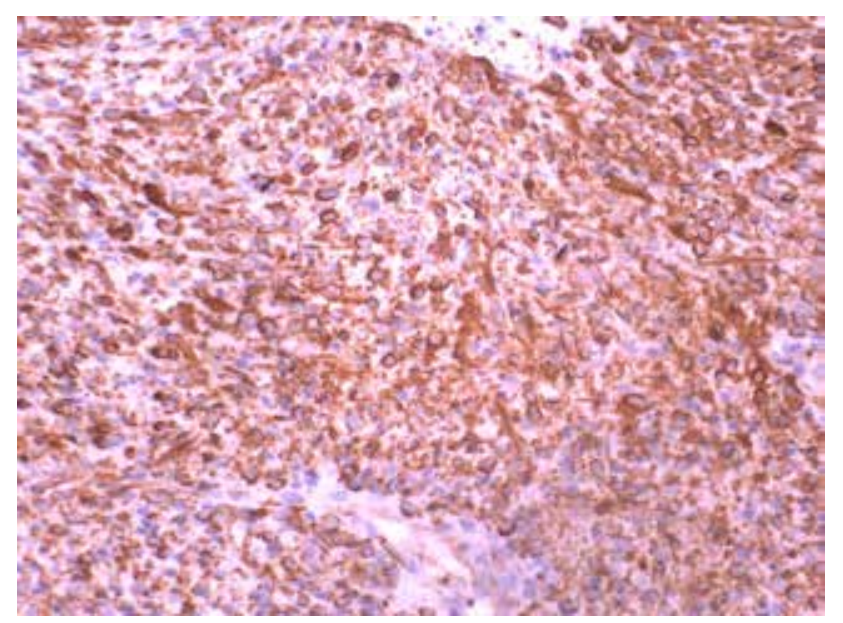

Figure 3: h-Caldesmon expression in tumour cells (IHC stain; X100).

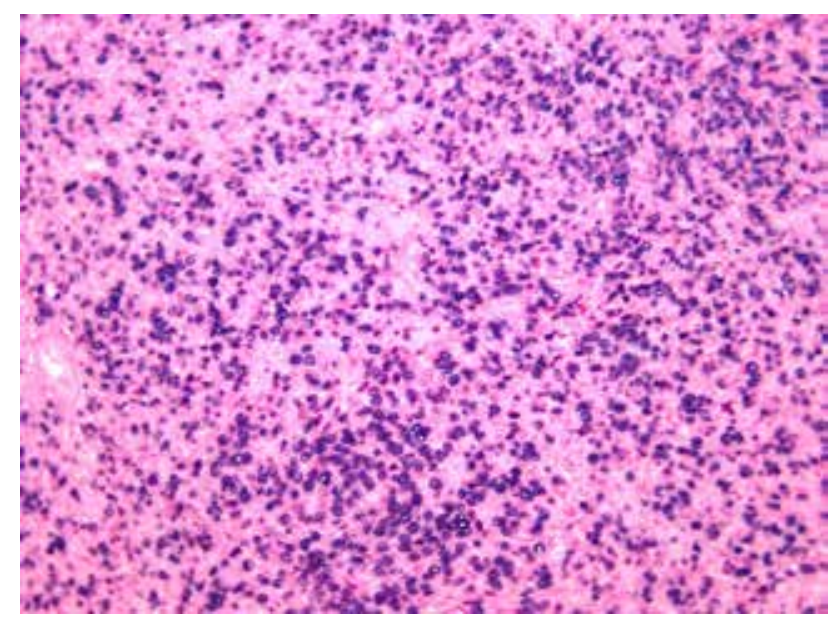

Figure 4: EBV in situ hybridisation displaying positive nuclear signal within tumour cells (EBV ISH; X200).

\section{Treatment}

The treatment modalities in this series included a combination of antiretroviral therapy (10 patients), surgical resection (5 patients), radiation ( 2 paediatric) or palliative therapy ( 2 patients). The survival outcome data of 9 patients was available as shown in Table 1.

\section{Discussion}

EBV-SMT are rare tumours that occur in immuocompromised patients. ${ }^{5,6}$ The rarity of this neoplasm is underscored by the presence of $14 \mathrm{HIV}$-related EBV-SMT that were histopathologically confirmed, during a five-year study time frame, at the largest hospital in Africa that has a high seroprevalence of HIV.

In this series, the tumours manifested predominantly in female patients $(77 \%)$ in topographic regions other than the female genital tract and/or breast, analogous to that documented by Hussein et al. ${ }^{6}$ Depending on the topographic site of involvement, the presenting symptoms included a noticeable mass lesion, pain, weakness of limbs, seizures or jaundice. Diagnostic clues to the presence of HIV-related EBV-SMT included a compromised immunological status, the presence of multifocal tumours, smooth muscle morphology, primitive round cell features and an admixture of lymphocytes. The lymphocytic component may at times be a subtle feature.

Morphological diversity within HIV-related EBV-SMT may manifest as hyper- and/or hypocellular smooth muscle fascicles, primitive round cell areas and prominent intratumoral oedema. An absence of desmin immunoexpression in this neoplasm should not deter pathologists from this diagnosis. Instead, round cell features in association with a desmin-negative scenario should prompt the use of smooth muscle lineage-specific immunohistochemistry, such as alpha SMA and/or h-Caldesmon. Notably, the EBV-positive myopericytoma may lack desmin expression. ${ }^{10-13}$ Primitive round cell morphology was suggested to represent a form of histological progression without an impact on prognosis. ${ }^{14}$ The tumours of three female patients ( 2 paediatric and 1 adult) herein demonstrated round cell features and their survival outcome was variable. One child survived for 30 months following radiation treatment, the remaining child is currently well following ophthalmic surgery and the adult patient remained paraplegic after surgical treatment.

The pathogenesis of EBV-SMT has been suggested to entail infection and transformation of smooth muscle cells by EBV with subsequent clonal expansion. Based on real-time polymerase chain analysis of the long terminal repeat region of the virus in 5 cases of EBV-SMT, Deyrup et al. demonstrated that multiple tumours in a patient derived from a separate clone of infected cells and concluded that these likely represented separate infection events. ${ }^{14}$

The differential diagnosis of EBV-SMT includes neoplastic and non-neoplastic spindle cell proliferations such as leiomyoma, leiomyosarcoma, meningioma, haemangiopericytoma, peripheral nerve sheath tumours, Kaposi sarcoma and mycobacterial or fungal pseudotumours. EBV in situ hybridisation plays an invaluable confirmatory role in the diagnosis of EBV-SMT.

Treatment modalities for HIV-related EBV-SMT include antiretroviral therapy, surgical resection, radiation and/or palliative care. The mammalian target of rapamycin (mTOR) 
pathway plays a regulating role in cell growth and proliferation. Inhibitors of this pathway, such as sirolimus, appear to be a promising treatment option for EBV-SMT. ${ }^{15}$ However, data about this therapeutic option for HIV-related EBV-SMT is limited..$^{16}$ Poor tolerance and resistance to cytotoxic chemotherapy are noteworthy findings during the treatment of EBV-SMT. ${ }^{17}$

While prolonged survival may occur in paediatric patients who have HIV-related EBV-SMT, AIDS-related co-morbidities are likely to contribute to mortality. As paraspinal lesions were common in this series, EBV-SMT should be included in the differential diagnosis of causes of spinal compression manifesting in HIVseropositive patients.

\section{Conclusion}

HIV-associated EBV-SMT are rare tumours that may develop in paediatric or adult patients. A female predominance and multifocal topographic involvement may be evident. AIDSrelated co-morbidities are likely to contribute to mortality; and, when these tumours occur in paraspinal regions debilitating neurological morbidity may manifest.

Conflict of interest - None declared by the authors.

Ethical clearance - Ethical approval of this study was obtained from the Human Research Ethics Committee (medical division) at the University of the Witwatersrand (clearance certificate number M150158).

Acknowledgements - We sincerely acknowledge our patients at $\mathrm{CHBAH}$. Mr E Liebenberg provided assistance with photography.

Funding - SP received support from SATBAT and the Fogarty International Centre [grant number 3U2RTW007370-05S1].

\section{References}

1. Ok CY, Li L, Young KH. EBV-driven B-cell lymphoproliferative disorders: from biology, classification and differential diagnosis to clinical management. Exp Mol Med. 2015;47:e132. doi:10.1038/ emm.2014.82.

2. Delecluse H-J, Feederle R, O'Sullivan B, et al. Epstein-Barr virusassociated tumours: an update for the attention of the working pathologist. J Clin Pathol. 2007;60:1358-64. doi:10.1136/ jcp.2006.044586.

3. Dekate J, Chetty R. Epstein-Barr virus-associated smooth muscle tumor. Arch Pathol Lab. 2016;140:718-22. https://doi.org/10.5858/ arpa.2015-0120-RS

4. McClain KL, Leach CT, Jenson HB, et al. Association of Epstein-Barr virus with leiomyosarcomas in young people with AIDS. N Engl J Med. 1995;332:12-8. https://doi.org/10.1056/NEJM199501053320103
5. Purgina B, Rao UNM, Miettinen M, et al. AIDS-related EBV-associated smooth muscle tumors: review of 64 published cases. Patholog Res Int 2011;10:1-10. doi:10.4061/2011/561548.

6. Hussein K, Rath B, Ludewig B, et al. Clinico-pathological characteristics of different types of immunodeficiency-associated smooth muscle tumours. Eur J Cancer. 2014;50:2417-24. https://doi.org/10.1016/j. ejca.2014.06.006

7. Rougemont AL, Alfieri $C$, Fabre $M$, et al. Atypical Epstein-Barr virus (EBV) latent protein expression in EBV-associated smooth muscle tumours occurring in paediatric transplant recipients. Histopathology. 2008;53:363-7. https://doi.org/10.1111/his.2008.53.issue-3

8. Ramdial PK, Sing Y, Deonarain J, et al. Dermal Epstein Barr virusassociated leiomyosarcoma: tocsin of acquired immunodeficiency syndrome in two children. Am J Dermatopathol. 2011;33:392-6. https://doi.org/10.1097/DAD.0b013e3181e5d16a

9. Ibebuike $\mathrm{KE}$, Pather $\mathrm{S}$, Emereole $\mathrm{O}$, et al. Epstein-Barr virus-associated smooth muscle tumour presenting as a parasagittal brain tumour. J Clin Neurosci. 2012;19:1589-91. https://doi.org/10.1016/j.jocn.2011.09.037

10. Ramdial PK, Sing Y, Deonarain J, et al. Extra-uterine myoid tumours in patients with acquired immunodeficiency syndrome: a clinicopathological reappraisal. Histopathology 2011;59: 1122-34. doi:10.1111/j.1365-2559.2011.04049.x.

11. Ramdial PK, Sing Y, Deonarain J, Singh B, Allopi L, Moodley P. Periampullary Epstein-Barr virus-associated myopericytoma. Hum Pathol 2011;42: 1348-54. doi:10.1016/j.humpath.2010.12.001.

12. Khaba MC, Ramdial PK, Pillay B, Steyn AJ, Nargan K. Epstein-Barr virus-associated myoid tumors in human immunodeficiency virusinfected patients. Journal of AIDS \& Clinical Research 2016;7:616. doi:10.4172/2155-6113.1000616.

13. Calderaro J, Polivka M, Gallien S, Bertheau P, Thiebault JB, et al. Multifocal Epstein-Barr virus (EBV)-associated myopericytoma in a patient with AIDS. Neuropathol Appl Neurobiol 2008;34: 115-7.

14. Deyrup AT, Lee VK, Hill CE, et al. Epstein-Barr virus-associated smooth muscle tumors are distinctive mesenchymal tumors reflecting multiple infection events. Am J Surg Pathol. 2006;30:75-82. https://doi.org/10.1097/01.pas.0000178088.69394.7b

15. Tan CS, Loh HL, Foo MW, et al. Epstein-Barr virus-associated smooth muscle tumors after kidney transplantation: treatment and outcomes in a single center. Clin Transplant. 2013;27:E462-8. doi:10.1111/ ctr.12139.

16. Shen Q, Feng W, Long MS, et al. Multicentric hepatic EBV-associated smooth muscle tumors in an AIDS patient: a case report, investigation of mTOR activation and review of the literature. Int J Clin Exp Pathol. 2011;30(4):421-9.

17. Ong KW, Teo $M$, Lee $V$, et al. Expression of EBV latent antigens, mammalian target of rapamycin, and tumor suppression genes in EBV-positive smooth muscle tumors: clinical and therapeutic implications. Clin Cancer Res. 2009;15:5350-8. https://doi. org/10.1158/1078-0432.CCR-08-2979

Received: 15-09-2016 Accepted: 17-05-2017 